\title{
A NOTE ON HAMMERSLEY'S INEQUALITY FOR ESTIMATING THE NORMAL INTEGER MEAN
}

\author{
RASUL A. KHAN
}

Received 7 August 2002

\begin{abstract}
Let $X_{1}, X_{2}, \ldots, X_{n}$ be a random sample from a normal $N\left(\theta, \sigma^{2}\right)$ distribution with an unknown mean $\theta=0, \pm 1, \pm 2, \ldots$. Hammersley (1950) proposed the maximum likelihood estimator (MLE) $d=\left[\bar{X}_{n}\right]$, nearest integer to the sample mean, as an unbiased estimator of $\theta$ and extended the Cramér-Rao inequality. The Hammersley lower bound for the variance of any unbiased estimator of $\theta$ is significantly improved, and the asymptotic (as $n \rightarrow \infty$ ) limit of Fraser-Guttman-Bhattacharyya bounds is also determined. A limiting property of a suitable distance is used to give some plausible explanations why such bounds cannot be attained. An almost uniformly minimum variance unbiased (UMVU) like property of $d$ is exhibited.
\end{abstract}

2000 Mathematics Subject Classification: 62F10, 62F12.

1. Introduction. Let $X_{1}, X_{2}, \ldots, X_{n}$ be a random sample from a normal $N(\theta$, $\sigma^{2}$ ) distribution with an unknown integer mean $\theta=0, \pm 1, \pm 2, \ldots$. Hammersley [3] proposed the maximum likelihood estimator (MLE) $d=\left[\bar{X}_{n}\right]$, nearest integer to the sample mean, as an unbiased estimator of $\theta$. He extended the Cramér-Rao variance inequality for any unbiased estimator $t=t\left(X_{1}, \ldots, X_{n}\right)$ and showed that

$$
\sigma_{t}^{2}(\theta) \geq \frac{1}{\exp \left(n / \sigma^{2}\right)-1}=H
$$

He noticed that $d$ does not achieve this bound (even asymptotically) and raised the question whether $H$ is a greatest lower bound, that is, if there is any estimator attaining this bound. The bound $H$ is improved to the extent that the new bound is almost twice the size of $H$. However, even the improved bound cannot be attained. The asymptotic limit (as $n \rightarrow \infty$ ) of Fraser-Guttman-Bhattacharyya bounds is also determined although $d$ still fails to achieve the asymptotic limit. We consider a suitable distance and use its limiting property to shed some light on the reasons why such bounds are unattainable. An intriguing behavior of $d$ is observed as follows. Define the loss functions

$$
L_{k}(\delta, \theta)=|\delta-\theta|^{k} I\{\delta \neq \theta\}, \quad k=0,1,2,
$$


where $I$ is the usual indicator function. Let $R_{k}(n)=E_{\theta} L_{k}(d, \theta)$ be the risk functions of $d$ when $k=0,1,2$. Then

$$
R_{k}(n) \sim \sqrt{\frac{8 \sigma^{2}}{n \pi}} \exp \left(-\frac{n}{8 \sigma^{2}}\right) \text { as } n \rightarrow \infty, k=0,1,2 .
$$

Hammersley [3] proved (1.3) when $k=2$. The interesting observation is that $R_{k}(n)$ has the same asymptotic behavior when $k=0,1$. In Section 2, we prove (1.3), improve the bound (1.1), and also determine the asymptotic limit of Bhattacharyya bounds. Also, we use a suitable distance and its limiting property to show the reason why such bounds cannot be attained even asymptotically. Such apparent anomalies seem to stem from the restricted parameter space. Some other properties (such as admissibility and minimaxity etc.) of $d$ have been explored by Khan [4, 5, 6], Ghosh and Meeden [2], and Kojima et al. [7]. However, the main focus of this paper is to settle some of the questions raised by Hammersley [3] himself regarding his bound, its attainment, and its relevance to his estimator. His problem is revisited for theoretical interest. A by-product of this pursuit is the conclusive observation that $d=\left[\bar{X}_{n}\right]$ is the best estimator even though $d$ fails to achieve any bound. In fact, at the end of Section 2, we show that $d$ is almost like uniformly minimum variance unbiased (UMVU) estimator in a restricted sense.

2. The main results. In what follows, $P_{\theta}$ denotes the probability under $\theta$ and $E_{\theta}$ denotes the corresponding expectation $\left(P_{i}\right.$ and $E_{i}$ have similar meanings). Let $\Phi(x)$ be the standard normal distribution function, and $\phi(x)=$ $(2 \pi)^{-1 / 2} \exp \left(-x^{2} / 2\right)$. The nearest integer to $y$ is denoted by $[y]$ throughout the paper without any further mention. It follows from the definition of $d=\left[\bar{X}_{n}\right]$ that

$$
\begin{aligned}
P_{i}(d-i=j)=P_{0}(d=j)= & \Phi\left(\frac{\sqrt{n}(j+1 / 2)}{\sigma}\right) \\
& -\Phi\left(\frac{\sqrt{n}(j-1 / 2)}{\sigma}\right)=f(j), \quad j=0, \pm 1, \pm 2, \ldots .
\end{aligned}
$$

Clearly, $f(-j)=f(j)$ with maximum at $j=0$, and

$$
\begin{aligned}
P_{i}(\text { error }) & =P_{i}(d \neq i)=E_{i} L_{0}(d, i)=R_{0}(n)=1-P_{i}(d=i) \\
& =1-f(0)=2\left(1-\Phi\left(\frac{\sqrt{n}}{\sigma}\right)\right) .
\end{aligned}
$$

Moreover, for $k=1,2$, we have

$$
\begin{aligned}
R_{k}(d, i) & =E_{i} L_{k}(d, i)=2 \sum_{j=1}^{\infty} j^{k} f(j)=R_{k}(n) \\
& =2 \sum_{j=1}^{\infty} j^{k}\left(\Phi\left(\frac{\sqrt{n}(j+1 / 2)}{\sigma}\right)-\Phi\left(\frac{\sqrt{n}(j-1 / 2)}{\sigma}\right)\right) .
\end{aligned}
$$


Since $d$ is integer-valued, hence

$$
R_{0}(n)=2\left(1-\Phi\left(\frac{\sqrt{n}}{2 \sigma}\right)\right) \leq R_{1}(n)=E_{i}|d-i| \leq E_{0} d^{2}=\sigma_{d}^{2}=R_{2}(n)
$$

Since $1-\Phi(x) \sim x^{-1} \phi(x)$ as $x \rightarrow \infty$, (2.2) implies (1.3) when $k=0$. The case $k=2$ in the asymptotic (1.3) was shown by Hammersley [3]; hence, (2.4) implies (1.3) when $k=1$.

We now consider the problem of improving the bound (1.1). Let $X_{1}, X_{2}, \ldots, X_{n}$ be i.i.d. $N\left(\theta, \sigma^{2}\right)$, where $\theta$ is not necessarily integer, and let $f_{n}(\theta)$ be the joint density of $\left(X_{1}, \ldots, X_{n}\right)$. Letting $T=\sum_{1}^{n} X_{i}$, we have

$$
f_{n}(\theta)=\exp \left(\frac{\theta T}{\sigma^{2}}-\frac{n \theta^{2}}{2 \sigma^{2}}\right) g\left(X_{1}, \ldots, X_{n}\right),
$$

where

$$
g\left(X_{1}, \ldots, X_{n}\right)=(2 \pi)^{-n / 2} \sigma^{-n} \exp \left(-\sum_{1}^{n} \frac{X_{i}^{2}}{2 \sigma^{2}}\right) .
$$

It is easy to verify that

$$
E_{\theta} \frac{f_{n}\left(\theta_{1}\right) f_{n}\left(\theta_{2}\right)}{f_{n}^{2}(\theta)}=\exp \left(\frac{n\left(\theta_{1} \theta_{2}+\theta^{2}-\theta\left(\theta_{1}+\theta_{2}\right)\right)}{\sigma^{2}}\right) .
$$

Let $t\left(X_{1}, \ldots, X_{n}\right)$ be an unbiased estimator of $\theta$, and let $h \neq 0$ ( $h$ is an integer if $\theta$ is an integer). Then, $E_{\theta} t\left(X_{1}, \ldots, X_{n}\right)=\theta$ implies that

$$
E_{\theta} t\left(X_{1}, \ldots, X_{n}\right) \frac{f_{n}(\theta \pm h)}{f_{n}(\theta)}=\theta \pm h, \quad E_{\theta} t\left(X_{1}, \ldots, X_{n}\right) S=2 h,
$$

where $S=\left(f_{n}(\theta+h)-f_{n}(\theta-h)\right) / f_{n}(\theta)$.

Since $E_{\theta} S=0$, (2.8) implies that $\operatorname{cov}_{\theta}(t, S)=2 h$. Consequently, CauchySchwarz inequality gives

$$
\sigma_{t}^{2}(\theta) \geq \frac{4 h^{2}}{E_{\theta} S^{2}}
$$

Using (2.7), we obtain

$$
\begin{aligned}
E_{\theta} S^{2} & =E_{\theta} \frac{f_{n}^{2}(\theta+h)}{f_{n}^{2}(\theta)}+E_{\theta} \frac{f_{n}^{2}(\theta-h)}{f_{n}^{2}(\theta)}-2 E_{\theta} \frac{f_{n}(\theta+h) f_{n}(\theta-h)}{f_{n}^{2}(\theta)} \\
& =\exp \left(\frac{n h^{2}}{\sigma^{2}}\right)+\exp \left(\frac{n h^{2}}{\sigma^{2}}\right)-2 \exp \left(-\frac{n h^{2}}{\sigma^{2}}\right) \\
& =2\left(\exp \left(\frac{n h^{2}}{\sigma^{2}}\right)-\exp \left(-\frac{n h^{2}}{\sigma^{2}}\right)\right) .
\end{aligned}
$$

Thus

$$
\sigma_{t}^{2}(\theta) \geq \frac{2 h^{2}}{\left(\exp \left(n h^{2} / \sigma^{2}\right)-\exp \left(-n h^{2} / \sigma^{2}\right)\right)}=B_{1}(h)
$$


It is easily seen that $\lim _{h \rightarrow 0} B_{1}(h)=\sigma^{2} / n$ (the usual bound). However, in our case under consideration, $\theta$ is an integer so that $h \neq 0$ must be an integer to make (2.8) and subsequent equations valid. Hence, maximizing the bound $B_{1}(h)$ over integers $h \neq 0$, we obtain

$$
\sigma_{t}^{2}(\theta) \geq \frac{2}{\exp \left(n / \sigma^{2}\right)-\exp \left(-n / \sigma^{2}\right)}=B
$$

It is easy to see that $B>H$ and $B / H \rightarrow 2$ as $n \rightarrow \infty$. Thus, the bound in (1.1) is not the best possible and there can be no estimator achieving (1.1) even asymptotically, and the question raised by Hammersley [3] is resolved.

Motivated by the preceding improvement, it is tempting to determine the limit of the $k$ th Fraser-Guttman-Bhattacharyya bound $B_{k}$ as $k \rightarrow \infty$. For integers $h \neq 0$, define the generalized difference operator (cf. Feller [1, page 220]) by

$$
S_{m}^{*}=\Delta_{h}^{m} f_{n}(\theta)=h^{-m} \sum_{i=0}^{m}\left(\begin{array}{c}
m \\
i
\end{array}\right)(-1)^{m+i} f_{n}(\theta+i h) .
$$

Let $S_{m}=S_{m}^{*} / f_{n}(\theta), m=1,2, \ldots$. Since $\sum_{i=0}^{m}(-1)^{m+i}\left(\begin{array}{c}m \\ i\end{array}\right)=0$, we note that $E_{\theta} S_{m}=0$, and

$$
\begin{aligned}
h^{m+k} & \operatorname{cov}_{\theta}\left(S_{m}, S_{k}\right) \\
& =\sum_{i=0}^{m} \sum_{j=0}^{k}\left(\begin{array}{c}
m \\
i
\end{array}\right)\left(\begin{array}{c}
k \\
j
\end{array}\right)(-1)^{m+k+i+j} \operatorname{cov}_{\theta}\left(\frac{f_{n}(\theta+i h)}{f_{n}(\theta)}, \frac{f_{n}(\theta+j h)}{f_{n}(\theta)}\right) .
\end{aligned}
$$

Using (2.7), one verifies that

$$
\begin{aligned}
\operatorname{cov}_{\theta} & \left(\frac{f_{n}(\theta+i h)}{f_{n}(\theta)}, \frac{f_{n}(\theta+j h)}{f_{n}(\theta)}\right) \\
& =E_{\theta}\left(\frac{f_{n}(\theta+i h)}{f_{n}(\theta)}-1\right)\left(\frac{f_{n}(\theta+j h)}{f_{n}(\theta)}-1\right) \\
& =E_{\theta} \frac{\left(f_{n}(\theta+i h) f_{n}(\theta+j h)-f_{n}(\theta+i h) f_{n}(\theta)\right)}{f_{n}^{2}(\theta)} \\
& =\exp \left(\frac{n i j h^{2}}{\sigma^{2}}\right)-1 .
\end{aligned}
$$

Hence

$$
h^{m+k} \operatorname{cov}_{\theta}\left(S_{m}, S_{k}\right)=\sum_{i=1}^{m} \sum_{j=1}^{k}\left(\begin{array}{c}
m \\
i
\end{array}\right)\left(\begin{array}{c}
k \\
j
\end{array}\right)(-1)^{m+k+i+j}\left(\exp \left(\frac{n i j h^{2}}{\sigma^{2}}\right)-1\right) .
$$

Moreover, it is easy to check that for any unbiased estimator $t=t\left(X_{1}, \ldots, X_{n}\right)$ of $\theta$,

$$
E_{\theta} t S_{1}=1 \Longleftrightarrow E_{\theta}(t-\theta) S_{1}=1, \quad E_{\theta}(t-\theta) S_{1}=0 \quad \forall j \geq 2 .
$$


Thus, $\gamma^{\prime}=(1,0,0, \ldots, 0)$ is a vector of covariances between $t$ and $S_{1}, \ldots, S_{k}$. Let $\Sigma=\left(\operatorname{cov}_{\theta}\left(S_{m}, S_{m^{\prime}}\right)\right)_{k \times k}$ be the covariance matrix of $S_{1}, S_{2}, \ldots, S_{k}$. Then, the $k$ th Bhattachayyra bound is $B_{k}(h)=\gamma^{\prime} \Sigma^{-1} \gamma$. A general expression for $B_{k}(h)$ is intractable although its asymptotic limit is discussed below. But, we first evaluate $B_{2}(h)$. Letting $y=\exp \left(n h^{2} / \sigma^{2}\right)$, it is easily seen from (2.16) that

$$
\Sigma=\left[\begin{array}{cc}
\frac{(y-1)}{h^{2}} & \frac{(y-1)^{2}}{h^{3}} \\
\frac{(y-1)^{2}}{h^{3}} & \frac{(y-1)\left(y^{2}+2 y-1\right)}{h^{4}}
\end{array}\right]
$$

and $B_{2}(h)=\gamma^{\prime} \Sigma^{-1} \gamma=h^{2}\left(y^{2}+2 y-1\right) / y\left(y^{2}-1\right)$.

Maximizing $B_{2}(h)$ over integers, we obtain

$$
\sigma_{t}^{2}(\theta) \geq B_{2}=\frac{\exp \left(2 n / \sigma^{2}\right)+2 \exp \left(n / \sigma^{2}\right)-1}{\exp \left(n / \sigma^{2}\right)\left(\exp \left(2 n / \sigma^{2}\right)-1\right)}>H
$$

where $H$ is the bound in (1.1). However, $B_{2} \sim H \sim \exp \left(-n / \sigma^{2}\right)$ as $n \rightarrow \infty$. In fact, we will show that $B_{k} \sim \exp \left(-n / \sigma^{2}\right)$ (as $\left.n \rightarrow \infty\right)$ even when $k \rightarrow \infty$.

It is clear from (2.16) that $\operatorname{cov}_{\theta}\left(S_{m}, S_{k}\right)$ is a polynomial in $x=\exp (\lambda y)(\lambda=$ $\left.n / \sigma^{2}, y=h^{2}\right)$ of degree $m k$ with leading coefficient 1 . Therefore, asymptotically (as $n \rightarrow \infty$ ), $\operatorname{cov}_{\theta}\left(S_{m}, S_{k}\right) \sim x^{m k} / h^{m+k}$. Thus, in view of the asymptotic consideration, we replace $\Sigma$ by $A=\left(x^{\alpha \beta} / h^{\alpha+\beta}\right)_{k \times k}, \alpha, \beta=1,2, \ldots, k$, and the bound $B_{k}$ is replaced by $B_{k}^{*}=\gamma^{\prime} A^{-1} \gamma=\left|A_{11}\right| /|A|$, where $A_{11}$ is the $(1,1)$ cofactor of $A$. Note that $B_{k}(h)$ and $B_{k}^{*}(h)$ both depend on $n$, and $B_{k}(h) \sim B_{k}^{*}(h)$ as $n \rightarrow \infty$. Fortunately, the determinants $|A|$ and $\left|A_{11}\right|$ are related to Vandermonde determinant. Consider the $k \times k$ Vandermonde determinant whose $i$ th row is $\left(x_{1}^{i-1}, x_{2}^{i-1}, \ldots, x_{k}^{i-1}\right), i=1,2, \ldots, k$. Then, it is well known that the Vandermonde determinant $V\left(x_{1}, \ldots, x_{k}\right)$ is given by

$$
V\left(x_{1}, x_{2}, \ldots, x_{k}\right)=\prod_{1 \leq i<j \leq k}\left(x_{j}-x_{i}\right)
$$

Setting $x_{i}=x^{i} / h, i=1,2, \ldots, k$, it is easy to see that

$$
\begin{aligned}
|A| & =\frac{x^{k(k+1) / 2}}{h^{k+k(k+1) / 2}} V\left(x_{1}, \ldots, x_{k}\right) \\
& =\frac{x^{k(k+1) / 2}}{h^{k+k(k+1) / 2}} \prod_{1 \leq i<j \leq k}\left(\frac{x^{j}}{h}-\frac{x^{i}}{h}\right) \\
& =\frac{x^{k(k+1) / 2}}{h^{k+k(k+1) / 2+\left(\begin{array}{c}
k \\
2
\end{array}\right)}} \prod_{1 \leq i<j \leq k} x^{j}\left(1-x^{i-j}\right) \\
& =\frac{x^{k(k+1) / 2+\sum_{i=0}^{k-1} i(i+1)}}{h^{k+k(k+1) / 2+\left(\begin{array}{c}
k \\
2
\end{array}\right)}} \prod_{1 \leq i<j \leq k}\left(1-x^{-(j-i)}\right) .
\end{aligned}
$$


Setting $p(k)=\sum_{i=1}^{k} i^{2}=k(k+1)(2 k+1) / 6$ and noting that

$$
\prod_{1 \leq i<j \leq k}\left(1-x^{-(j-i)}\right)=\prod_{i=1}^{k-1}\left(1-x^{-i}\right)^{k-i},
$$

we obtain

$$
|A|=\frac{x^{p(k)}}{h^{k+k(k+1) / 2}+\left(\begin{array}{c}
k \\
2
\end{array}\right)} \prod_{i=1}^{k-1}\left(1-x^{-i}\right)^{k-i} .
$$

Next, we note that $A_{11}$ is $(k-1) \times(k-1)$ determinant given by

$$
\begin{aligned}
\left|A_{11}\right| & =\left|\begin{array}{cccc}
\frac{x^{4}}{h^{4}} & \frac{x^{6}}{h^{5}} & \ldots & \frac{x^{2 k}}{h^{k+2}} \\
\cdots & \cdots & \ldots & \cdots \\
\frac{x^{2 k}}{h^{k+2}} & \frac{x^{3 k}}{h^{k+3}} & \ldots & \frac{x^{k^{2}}}{h^{2 k}}
\end{array}\right| \\
& =\frac{x^{2 \sum_{j=2}^{k} j}}{h^{4+5+\cdots+(k+2)}} V\left(x_{1}, x_{2}, \ldots, x_{k-1}\right),
\end{aligned}
$$

where $x_{1}=x^{2} / h, x_{2}=x^{3} / h, \ldots, x_{k-1}=x^{k} / h$.

Since $4+5+\cdots+(k+2)=\sum_{i=2}^{k} i+2(k-1)=k(k+1) / 2+2 k-3$, we have

$$
\begin{aligned}
\left|A_{11}\right| & =\frac{x^{2 \sum_{j=2}^{k} j}}{h^{k(k+1) / 2+2 k-3+\left(\begin{array}{c}
k-1 \\
2
\end{array}\right)}} \prod_{1 \leq i<j \leq k}\left(x^{j+1}-x^{i+1}\right) \\
& =\frac{x^{2 \sum_{j=2}^{k} j+\sum_{i=1}^{k-2} i(i+2)}}{h^{k(k+1) / 2+2 k-3+\left(\begin{array}{c}
k-1 \\
2
\end{array}\right)}} \prod_{1 \leq i<j \leq k}\left(1-x^{-(j-i)}\right) .
\end{aligned}
$$

Also, since $2 \sum_{j=2}^{k} j+\sum_{i=1}^{k-2} i(i+2)=p(k)-1$, we obtain

$$
\left|A_{11}\right|=\frac{x^{p(k)-1}}{h^{k(k+1) / 2+2 k-3+\left(\begin{array}{c}
k-1 \\
2
\end{array}\right)}} \prod_{i=1}^{k-2}\left(1-x^{-i}\right)^{k-1-i} .
$$

Recalling the definition $x=\exp (\lambda y), \lambda=n / \sigma^{2}, y=h^{2}$, from (2.23) and (2.26), we have

$$
\begin{aligned}
B_{k}^{*}(y) & =\frac{\left|A_{11}\right|}{|A|}=\frac{h^{2}}{x \prod_{i=1}^{k-1}\left(1-x^{-i}\right)} \\
& =\frac{y}{\exp (\lambda y) \prod_{i=1}^{k-1}(1-\exp (-\lambda y i))} .
\end{aligned}
$$

Now, $B_{k}^{*}(y)$ is to be maximized over $y=h^{2}=1,4,9, \ldots$ First, consider $B_{2}^{*}(y)$. Obviously, $B_{2}^{*}(y)=y /(\exp (\lambda y)-1)$ is decreasing in $y$, hence $\max _{y \geq 1} B_{2}^{*}(y)=$ $B_{2}^{*}(1)$. 
Moreover, we note that

$$
B_{k+1}^{*}(y)=\frac{B_{k}^{*}(y)}{1-\exp (-\lambda k y)},
$$

and by induction, it is easy to see that $B_{k}^{*}(y)$ is decreasing in $y$. Thus

$$
\max _{k \geq 1} B_{k}^{*}(y)=B_{k}^{*}(1)=\frac{\exp (-\lambda)}{\prod_{i=1}^{k-1}(1-\exp (-\lambda i))}, \quad \lambda=\frac{n}{\sigma^{2}} .
$$

It is easy to verify that $\prod_{i=1}^{\infty}(1-\exp (-\lambda i))$ is convergent, and hence

$$
\lim _{k \rightarrow \infty} B_{k}^{*}(1)=\frac{\exp \left(-n / \sigma^{2}\right)}{\prod_{i=1}^{\infty}\left(1-\exp \left(-n i / \sigma^{2}\right)\right)}
$$

Clearly, $B_{k}^{*}(1)$ and $\lim _{k \rightarrow \infty} B_{k}^{*}(1)$ are both similar to $\exp \left(-n / \sigma^{2}\right) \sim H$ (as $n \rightarrow$ $\infty)$, and hence the asymptotic limit of Bhattacharyya bounds does not improve the Hammersley bound.

Now, the question arises: why such bounds are unattainable? To see the reason, we define a suitable distance and examine its limit. Let $f_{n}(\theta)$ be the joint density of $\left(X_{1}, \ldots, X_{n}\right)$ under $P_{\theta}$ relative to a $\sigma$-finite measure $\mu_{n}$, where $\theta \in \Omega$ (not necessarily normal). Let $\theta_{1}, \theta_{2} \in \Omega$, and define

$$
\begin{gathered}
D_{n}=\int\left|f_{n}\left(\theta_{1}\right)-f_{n}\left(\theta_{2}\right)\right| d \mu_{n}, \\
\rho_{n}\left(\theta_{1}, \theta_{2}\right)=\int \sqrt{f_{n}\left(\theta_{1}\right) f_{n}\left(\theta_{2}\right)} d \mu_{n} .
\end{gathered}
$$

We need the following elementary lemma of independent and general interest.

LEMMA 2.1. The upper and lower bounds for $D_{n}$ are given by $2\left(1-\rho_{n}\left(\theta_{1}, \theta_{2}\right)\right)$ $\leq D_{n} \leq 2 \sqrt{1-\rho_{n}^{2}\left(\theta_{1}, \theta_{2}\right)}$.

Proof. Clearly,

$$
\begin{aligned}
D_{n} & =E_{\theta_{1}}\left|1+\sqrt{\frac{f_{n}\left(\theta_{2}\right)}{f_{n}\left(\theta_{1}\right)}}\right|\left|1-\sqrt{\frac{f_{n}\left(\theta_{2}\right)}{f_{n}\left(\theta_{1}\right)}}\right| \\
& \geq E_{\theta_{1}}\left(1-\sqrt{\frac{f_{n}\left(\theta_{2}\right)}{f_{n}\left(\theta_{1}\right)}}\right)^{2}=2\left(1-\rho_{n}\left(\theta_{1}, \theta_{2}\right)\right),
\end{aligned}
$$

proving the first half of the inequality. Moreover, by Cauchy-Schwarz inequality, we have

$$
\begin{aligned}
D_{n}^{2} & \leq E_{\theta_{1}}\left(1+\sqrt{\frac{f_{n}\left(\theta_{2}\right)}{f_{n}\left(\theta_{1}\right)}}\right)^{2} E_{\theta_{1}}\left(1-\sqrt{\frac{f_{n}\left(\theta_{2}\right)}{f_{n}\left(\theta_{1}\right)}}\right)^{2} \\
& =2\left(1+\rho_{n}\left(\theta_{1}, \theta_{2}\right)\right) \cdot 2\left(1-\rho_{n}\left(\theta_{1}, \theta_{2}\right)\right),
\end{aligned}
$$

and the lemma is proved. 
In the normal case $N\left(\theta, \sigma^{2}\right)$, recall the density (2.5) and note that

$$
\sqrt{f_{n}\left(\theta_{1}\right) f_{n}\left(\theta_{2}\right)}=f_{n}\left(\frac{\theta_{1}+\theta_{2}}{2}\right) \exp \left(-\frac{n\left(\theta_{1}-\theta_{2}\right)^{2}}{8 \sigma^{2}}\right),
$$

and hence $\rho_{n}\left(\theta_{1}, \theta_{2}\right)=\rho^{n}=\exp \left(-n\left(\theta_{1}-\theta_{2}\right)^{2} / 8 \sigma^{2}\right)$.

Let $\theta_{1} \neq \theta_{2}$, and set $h=\theta_{1}-\theta_{2}$. Then, the inequality in Lemma 2.1 becomes

$$
2\left(1-\exp \left(-\frac{n h^{2}}{8 \sigma^{2}}\right)\right) \leq D_{n} \leq 2 \sqrt{1-\exp \left(-\frac{n h^{2}}{8 \sigma^{2}}\right)}, \quad \lim _{n \rightarrow \infty} D_{n}=2 .
$$

However, in the process of using Cauchy-Schwarz inequality to obtain Hammersley's bound (1.1) (although there is no escape from it), the following inequality occurs in disguise:

$$
D_{n}^{2} \leq E_{\theta_{1}}\left(1-\frac{f_{n}\left(\theta_{2}\right)}{f_{n}\left(\theta_{1}\right)}\right)^{2}=\exp \left(\frac{n h^{2}}{\sigma^{2}}\right)-1 .
$$

This very weak inequality is the cause of poor lower bound when $\theta$ is restricted to integers ( $h \neq 0$ is restricted to integers as well).

Now, we compute $D_{n}$ exactly in the normal case. First, suppose that $\theta_{2}>\theta_{1}$. Letting $h=\theta_{2}-\theta_{1}>0$, we have

$$
R_{n}=\frac{f_{n}\left(\theta_{2}\right)}{f_{n}\left(\theta_{1}\right)}=\exp \left(\frac{h S_{n}}{\sigma^{2}}-\frac{n h^{2}}{2 \sigma^{2}}\right)
$$

where $S_{n}=\sum_{1}^{n}\left(X_{i}-\theta_{1}\right) \sim N\left(0, n \sigma^{2}\right)$ under $P_{\theta_{1}}$. Since $R_{n} \geq 1 \Leftrightarrow S_{n} \geq n h / 2$, it is easy to verify that

$$
D_{n}=E_{\theta_{1}}\left|1-R_{n}\right|=4 \Phi\left(\frac{\sqrt{n} h}{2 \sigma}\right)-2 \text {. }
$$

In general, for any $\theta_{1} \neq \theta_{2}$ and $h=\theta_{2}-\theta_{1}$, we have

$$
\begin{gathered}
D_{n}=4 \Phi\left(\frac{\sqrt{n}|h|}{2 \sigma}\right)-2, \\
1-\frac{1}{2} D_{n} \sim \frac{1}{|h|} \sqrt{\frac{8 \sigma^{2}}{n \pi}} \exp \left(-\frac{n h^{2}}{8 \sigma^{2}}\right) \quad \text { as } n \rightarrow \infty .
\end{gathered}
$$

In particular, if $\theta$ is restricted to integers and $|h|=1$, then

$$
1-\frac{1}{2} D_{n}=2\left(1-\Phi\left(\frac{\sqrt{n}}{2 \sigma}\right)\right) \sim \sqrt{\frac{8 \sigma^{2}}{n \pi}} \exp \left(-\frac{n}{8 \sigma^{2}}\right) \text { as } n \longrightarrow \infty,
$$

which is the asymptotic (1.3) noted earlier. 
2.1. An almost UMVU property of $d$. It has been shown by Khan [5] that under squared-error loss function, $d=\left[\bar{X}_{n}\right]$ is admissible in the class of integervalued estimators $\mathscr{T}$, while Ghosh and Meeden [2] proved its admissibility in $\mathscr{I}$ under a more general loss function. It has been further observed by Khan [6] that $d$ is the best invariant estimator in the class $\mathscr{I}$ and that it is admissible under a generalized version of 0-1 loss function. Here, we show that $d$ is almost like UMVU estimator in $\Phi$. Thus, we conclude the discussion about Hammersley's estimator with the following interesting observation. It should be noted that the statistic $d_{1}=\bar{X}_{n}$ continues to be sufficient for $\theta \in \mathscr{L}=\{0, \pm 1, \pm 2, \ldots\}$ although it is not complete for the obvious reason that $d-d_{1}$ is unbiased estimator of 0 but $d_{1}-d \neq 0$ with positive probability. Moreover, $\sigma_{d}^{2}, \sigma_{d_{1}}^{2}$, and $\operatorname{cov}\left(d, d_{1}\right)$ are independent of $\theta$. This fact was exploited in Khan [4] to determine the best unbiased estimator $d_{\alpha}=\alpha d+(1-\alpha) d_{1}(0 \leq \alpha \leq 1)$ which strictly dominates $d$ and $d_{1}$. However, in view of sufficiency, one considers estimators of the form $t=t\left(\bar{X}_{n}\right)$, and we further restrict this class to integer-valued unbiased estimators of the form $T=f\left(\bar{X}_{n}\right)$, where $f$ is an integer-valued function on the real line. Also, it is logical to assume that $f(i)=i, i \in \mathscr{L}$, and $f$ is nondecreasing. Under these conditions, we will show that $\sigma_{f}^{2}(\theta) \geq \sigma_{d}^{2}(\theta)$ for all $\theta$ and for any unbiased $f\left(\bar{X}_{n}\right)$. Thus, $d$ is the best unbiased estimator in this class and this makes it almost like UMVU estimator. The proof of this elementary fact now follows. Let $K=\sqrt{n / 2 \pi \sigma^{2}}$, and let $f=f\left(\bar{X}_{n}\right)$, where $f$ is integer-valued with the above properties. It then follows from the assumed unbiasedness of $f$ that

$$
\begin{aligned}
\sigma_{f}^{2} & =K \int_{-\infty}^{\infty}(f(y)-i)^{2} \exp \left(-\frac{n}{2 \sigma^{2}}(y-i)^{2}\right) d y \\
& =K \int_{-\infty}^{\infty}(f(i+u)-i)^{2} \exp \left(-\frac{n u^{2}}{2 \sigma^{2}}\right) d u
\end{aligned}
$$

We note that $|f(i+u)-i|$ is minimized for all $i$ if and only if $f(u)=[u]$, that is, $\inf _{f}|f(i+u)-i|=|[u]|$. In fact, since $[u+i]=i+[u]$, letting $\eta=u-[u]$ $(|\eta| \leq 1 / 2)$, we see that if $|\eta|<1 / 2$, then

$$
|(f(i+u)-i)-[u]|=|f([i+u]+\eta)-[i+u]|=0
$$

if and only if $f(u)=[u]$. On the other hand, if $\eta= \pm 1 / 2$, then

$$
|(f(i+u)-i)-[u]|=|f([i+u]+\eta)-[u+i]|=1
$$

if and only if $f(u)=[u]$. Alternatively, we have

$$
|f(i+u)-i|=|f([i+u]+\eta)-i| \geq|f([i+u])-i|=|[u]|
$$


Hence, it follows from the above that

$$
\sigma_{f}^{2} \geq K \int_{-\infty}^{\infty}|[u]|^{2} \exp \left(-\frac{n u^{2}}{2 \sigma^{2}}\right) d u=\sigma_{d}^{2} .
$$

Thus, $d$ is the best unbiased estimator in the class of integer-valued estimators which are functions of sufficient statistic.

\section{REFERENCES}

[1] W. Feller, An Introduction to Probability Theory and Its Applications. Vol. II, John Wiley \& Sons, New York, 1966.

[2] M. Ghosh and G. Meeden, Admissibility of the MLE of the normal integer mean, Sankhyā Ser. B 40 (1978), no. 1-2, 1-10.

[3] J. M. Hammersley, On estimating restricted parameters, J. Roy. Statist. Soc. Ser. B 12 (1950), 192-240.

[4] R. A. Khan, On some properties of Hammersley's estimator of an integer mean, Ann. Statist. 1 (1973), 756-762.

[5] _ A note on the admissibility of Hammersley's estimator of an integer mean, Canad. J. Statist. 6 (1978), no. 1, 113-119.

[6] _ A note on Hammersley's estimator of an integer mean, J. Statist. Plann. Inference 88 (2000), no. 1, 37-45.

[7] Y. Kojima, H. Morimoto, and K. Takeuchi, Two "best" unbiased estimators of normal integral mean, Statistics and Probability: Essays in Honor of C. R. Rao (G. Kallianpur, P. R. Krishnaiah, and J. K. Ghosh, eds.), North-Holland, New York, 1982, pp. 429-441.

Rasul A. Khan: Department of Mathematics, Cleveland State University, 2121 Euclid Avenue, RT 1515, Cleveland, OH 44115, USA

E-mail address: khan@math.csuohio.edu 


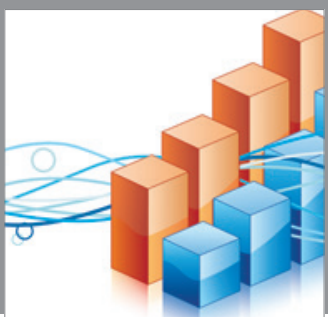

Advances in

Operations Research

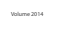

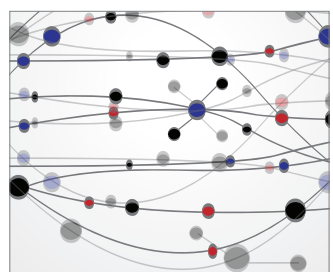

\section{The Scientific} World Journal
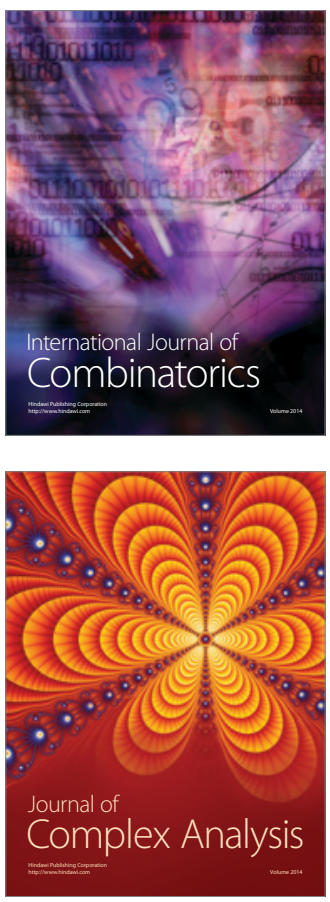

International Journal of

Mathematics and

Mathematical

Sciences
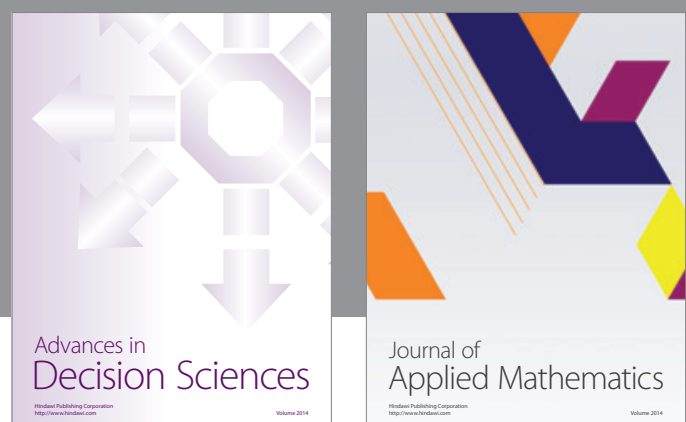

Journal of

Applied Mathematics
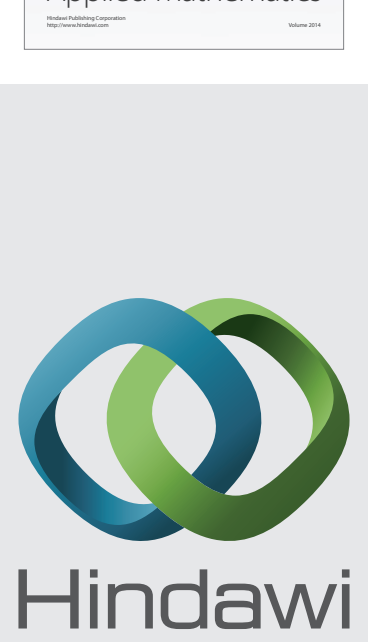

Submit your manuscripts at http://www.hindawi.com
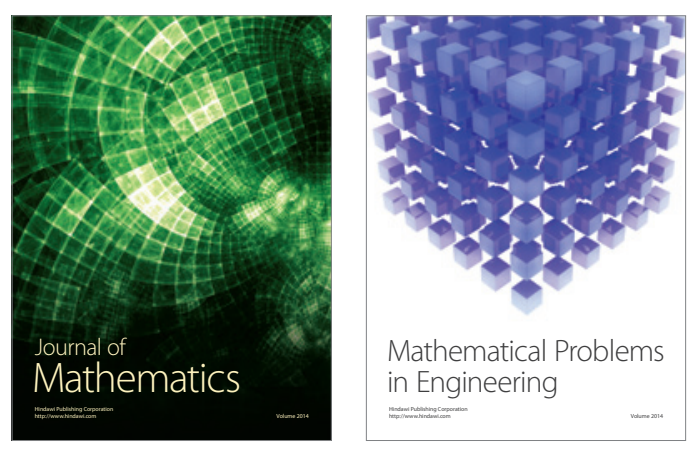

Mathematical Problems in Engineering
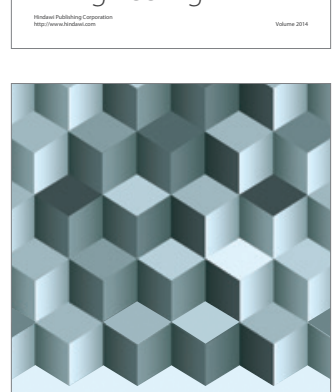

Journal of

Function Spaces
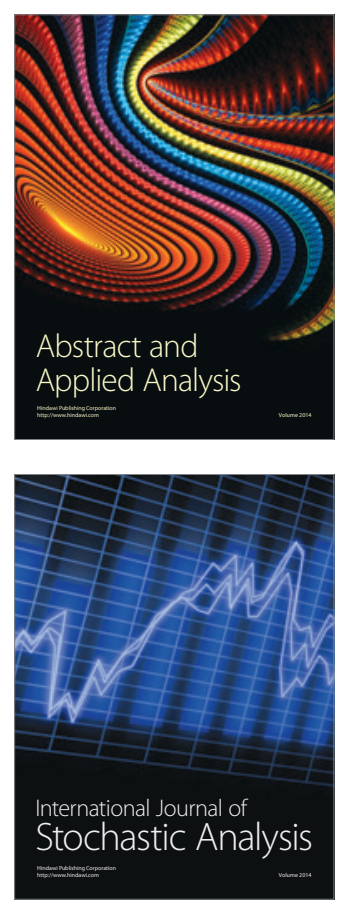

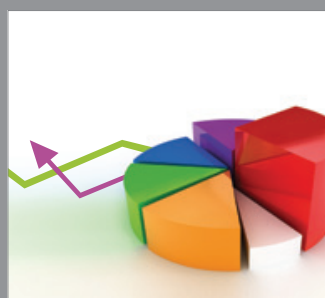

ournal of

Probability and Statistics

Promensencen
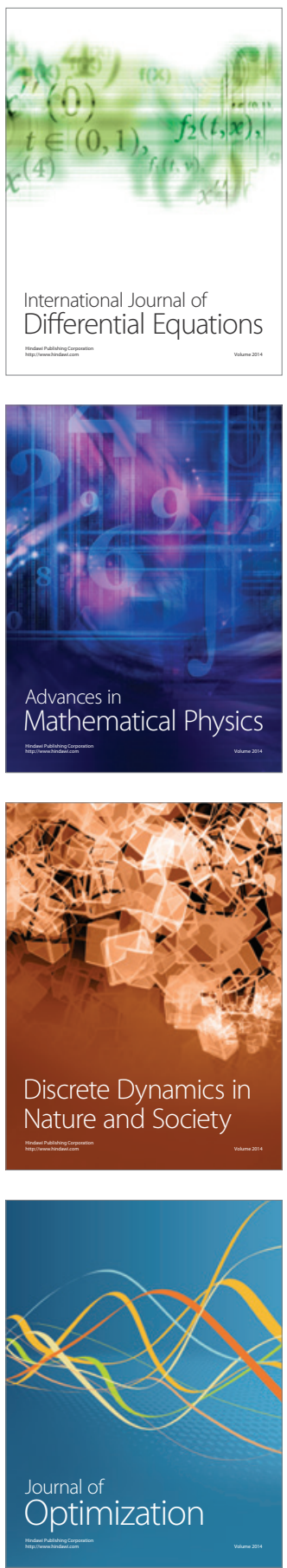